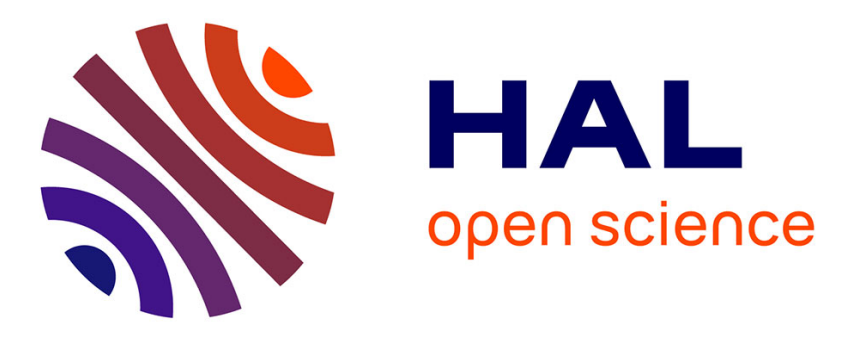

\title{
On Queue-Aware Power Control in Interfering Wireless Links: Heavy Traffic Asymptotic Modelling and Application in QoS Provisioning
}

Apostolos Destounis Destounis, Mohamad Assaad, Merouane Debbah, Bessem Sayadi, Afef Feki

\section{To cite this version:}

Apostolos Destounis Destounis, Mohamad Assaad, Merouane Debbah, Bessem Sayadi, Afef Feki. On Queue-Aware Power Control in Interfering Wireless Links: Heavy Traffic Asymptotic Modelling and Application in QoS Provisioning. IEEE Transactions on Mobile Computing, 2013, 13 (10), pp.2345 2356. 10.1109/TMC.2013.65 . hal-01098941

\section{HAL Id: hal-01098941 \\ https://hal.science/hal-01098941}

Submitted on 30 Dec 2014

HAL is a multi-disciplinary open access archive for the deposit and dissemination of scientific research documents, whether they are published or not. The documents may come from teaching and research institutions in France or abroad, or from public or private research centers.
L'archive ouverte pluridisciplinaire HAL, est destinée au dépôt et à la diffusion de documents scientifiques de niveau recherche, publiés ou non, émanant des établissements d'enseignement et de recherche français ou étrangers, des laboratoires publics ou privés. 


\title{
On Queue-Aware Power Control in Interfering Wireless Links: Heavy Traffic Asymptotic Modelling and Application in QoS Provisioning
}

\author{
Apostolos Destounis, Student Member, IEEE, Mohamad Assaad, Member, IEEE, Mérouane Debbah, \\ Senior Member, IEEE Bessem Sayadi, Member, IEEE and Afef Feki Member, IEEE
}

\begin{abstract}
In this work we address the problem of power allocation for interfering transmitter-receiver pairs so that the probability that each queue length exceeds a specified threshold is fixed at a desired value. One application is satisfying QoS requirements in a dense cellular network. We deal with this problem using heavy traffic approximation techniques which lead to an asymptotic model of a (controlled) stochastic differential equation. The proposed power control strategy consists of allocating most of the power according to the states of the channel and a smaller fraction according to the queue lengths, for which we find a closed-form expression. We first consider a scenario where all channel realizations and queue lengths are known instantaneously to every transmitter. Then, the algorithm is extended to the case where only local SINR feedback is available and when queue length information is shared with delays among the transmitters. These models and results are also extended to the case where the transmitters are equipped with multiple antennas. Finally, the applicability in practical system settings are discussed and simulation results are provided to illustrate the performance of the proposed method.
\end{abstract}

\section{INTRODUCTION}

The explosive growth of video and data applications demands over wireless networks poses a serious challenge to mobile operators. A promising way to deal with this demand is the concept of Small Cell Networks, which are dense cellular networks with low power base stations and frequency reuse one [3]. One of the key issues arising in such networks that intercell interference is severe, as there are now many base stations close to each other. Cooperation between the base stations of a cellular network has been shown to mitigate the intercell interference and increase spectral efficiency [4], [5]. However in practice this is not always feasible due to

A. Destounis, B. Sayadi and A. Feki are with Alcatel-Lucent Bell Labs France, Villarceaux, Route de Villejust, 91620, Nozay, cedex. France (\{apostolos.destounis; bessem.sayadi; afef.feki\}@alcatel-lucent.com)

M. Assaad is with the Telecommunications Department at SUPÉLEC, 3 rue Joliot-Curie, 91192, Gif-sur-Yvette, cedex. France. (mohamad.assaad@supelec.fr)

M. Debbah is with the Alcatel-Lucent Chair on Flexible Radio at SUPÉLEC, 3 rue Joliot-Curie, 91192, Gif-sur-Yvette, cedex. France. (merouane.debbah@supelec.fr)

Parts of this paper have been presented in SPAWC 2012, Cesme, Turkey [1], the 28th Meeting of the WWRF, Piraeus, Greece [2] and the 2nd Bell Labs Science Workshop, Villarceaux, France 2011 limitations in backhaul capacity. Moreover, the incoming traffic at the base stations is not taken into account in these works. As in practical cases the traffic arrives with random patterns and the users have requirements in terms of Quality of Service (such as delays, bit error rates etc.), a better approach is to make the resource allocation at the base stations adapted to the real-time traffic characteristics and queue states.

In this paper we will consider a system with $K$ transmitters, each having $L$ antennas and operating in the same frequency band $W$, each of them serving one receiver. The additional challenge here is that links interfere with each other so the queue length of one transmitter depends also on the power (and beamforming) allocations of the other transmitters. This setting can correspond to small cells employing the same subcarriers to serve the users. The objective is to minimize the total power over an infinite time horizon such that the probability that the queue size at each transmitter exceeding a threshold is fixed (unlike the approach in the references cited where the objective is to minimize a single function of all the queue lengths). This goal may correspond to fixing a data loss probability (for finite buffers at the transmitters) in some desired values or some delay requirement, which is a crucial QoS aspect in multimedia applications. In delay-constrained cases we can argue that due to Little's law the (average) delay is proportional to the queue length therefore bounding the delay below a desired threshold is equivalent to bounding the queue length below a threshold corresponding to the delay bound. Since the incoming traffic and channels evolve randomly however, such a constraint is not possible to hold, thus we will consider the following probabilistic QoS metric for the queue length at each transmitter $k$

$$
\operatorname{Pr}\left\{q_{k}(t)>q_{k}^{\text {thr }}\right\}=\delta_{k},
$$

which means setting a buffer (equivalently, delay) outage probability in some values that can be tolerated by the application. In this work we tackle the problem by proposing a power control strategy so that these 
constraints are met, based on heavy traffic asymptotic modelling. The approach is then to divide the power into two parts: equilibrium and reserve powers. The equilibrium power problem consists in allocating the power according to the channel states so that on average the transmission rate is equal to the arrival rate. In the literature, this type of problems has been widely studied for both single and multiple antennas systems (one can refer to [6],[7] for more details). The main challenge in this paper is in the modelling and allocation of the reserve power. An immediate approach to optimally control the reserve power would be to formulate the problem using optimal control theory and HJB (HamiltonJacobi-Bellman) framework. However the constraint (1) makes the problem very hard and solutions are not even guaranteed to be tractable. In addition, the system (under some control policies) may not be ergodic. Even if the system is stationary and ergodic, finding a closed form expression of the stationary distribution function of the queue evolution is not easy. This is due to the interaction between the different users' queues through the interference. In this paper, we tackle the above problem as follows. We first show that the queues of the users in the heavy traffic regime can be modeled as a reflected multidimensional stochastic differential equation (SDE). Then, taking advantage of the specific structure of the reflection matrix, we propose a control policy that decouples the multidimensional SDE into several parallel SDEs and ensures that an invariant measure for each of these SDEs exists. Using results from probability theory, we can obtain a closed form expression of the stationary distribution function of the dynamics of each SDE which allows finding a relation between the reserve power allocated and the overflow probability in (1). Notice that the value of the reserve power allocated by our algorithm compared to the equilibrium power is very small (as we will show later in this paper). In other words, the sub optimality gap between our reserve power approach and other optimal control approach (e.g. using HJB) is small in many scenarios.

Regarding the problem of simultaneous transmissions over interfering channels, substantial work has been done in power allocation so that the Signal-to-Noise Ratio at each receiver is above a specified threshold. In [8] such an algorithm, which is totally distributed, has been proposed and in subsequent years modifications and extensions have been made; for an extensive survey of power control algorithms with target SNR refer to [6] and references therein. This approach however is not suited for the traffic nature of data and video streaming applications, as they do not adapt to the traffic, queue states and/or the specific requirements of the application requested. In this direction, in [9] a scheduler based on $H$-infinity control was proposed in order to regulate the buffers of small cell base stations around a target length. In [10], the problem of power control for VBR video streaming over a cellular network is concerned, with the assumption that the videos requested are stored at the base stations (therefore stochastic traffic dynamics are not taken into account). The authors investigate the problem of throughput maximization under overflow and underflow constraints at the receivers' playout buffers and propose an optimal centralized and a near optimal decentralized algorithm under some feasibility assumptions for the SINR.

The authors in [11] consider dynamic scheduling (inside the cell) and power allocation (for intercell interference mitigation) so that a function of the queue lengths that corresponds to the average delay in the system is minimized. The problem is formulated as a Markov Decision Problem, and an online learning algorithm is used for the solution. The proposed algorithm is semidecentralized in the sense that a central controller sets the transmission power levels but the scheduling decision is taken at each base station. For a survey of the use of such tools in resource allocation problems see [12] and references therein. However, in these problems the objective is to optimize a single objective function subject to constraints on the expected values of the queues, which are weaker then the overflow probabilities we consider here. In addition, these techniques requite the solution of the Bellman equation which in general can be solved off-line only numerically at a high computational cost, and learning algorithms for on-line implementation may converge slowly (especially when the number of users and queue capacity are large).

Another line of work regarding resource allocation in wireless networks is done by using Lyapunov drift techniques (see [13], [12] and references therein). These works address the problem of minimizing a cost function for the network while keeping the queues stable. However, in our work we are interested in satisfying individual QoS constraints for each user, in a form which is much stronger than requiring just stability of the queues. Finally, another approach is to convert the delay constraints into equivalent rate constraints, however queue lenght information is not taken into account and it works well for relatively large delays [12].

The approach followed in this paper is based on the heavy traffic asymptotic modelling of a network. Initially used for the analysis of queues and queuing networks, it consists of examining the system's behaviour as the arrival rates become almost as big as the service rates. It turns out that the models in this asymptotic case become more tractable to handle and their study can reveal useful information for the system's behaviour even when this condition does not hold. Also, due to its tractability, the heavy traffic asymptotic regime can be used to find analytically a control policy in the network (e.g. routing policy, transmission scheduling, service rate adjustment etc.); this policy then can be applied in nonasymptotic situations of the network, with some proper modifications (see [14] and references therein).

In the context of wireless communications, heavy traffic models have been used to analyze the performance of the MaxWeight and Exponential scheduling algorithm in 
[15] and [16] respectively. Moreover, authors in [17] have studied the performance of a throughput-optimal rate allocation in a two-user MIMO system (with a common channel for the users) when the incoming traffic is nearly equal to the rate of each user. This was generalized in [18] for multiple antennas at the transmitter, each serving one user. In both cases the behaviour of the queues turns out to be a multidimensional Brownian motion constrained in the positive orthant. Also, in [19], it is proved that for the latter case and time-varying channels the policy of assigning rates at the boundary of the capacity region is asymptotically optimal in the sense that it minimizes a weighted sum of packets in the queue in heavy traffic; moreover, this quantity is proven to be a reflecting Brownian motion with regime switching.

The first application of the heavy traffic approach to the power control problem was made by [20], where it was used to derive an optimal power control in the setting of a single base station serving many users via time-varying but orthogonal channels. In that work, each user is preassigned one channel and the authors assumed total power constraints and that power can be reallocated from one channel to another. The control policy was specified numerically. Simulation results of this policy can be found in [21]. Also, in [22] an optimal power control was derived for the point-to-point link over a fading channel. It was shown that the delayoptimal policy is a simple single-threshold one and simulations results show that the resulting cost is very close to the one obtained by solving the original control problem. In the latter three works, heavy traffic condition is imposed by preallocating suitable amount of power according to the channel state and then allocating a (much smaller) amount or reserve power according to channel states and queue lengths. Indeed, without this additional reserve power allocation the delay becomes unbounded [22], [23].

The main contributions of this paper are: $(i)$ Derivation of a heavy traffic asymptotic model for this system of $K$ interfering wireless links for transmitters equipped with single and multiple antennas, (ii) Derivation of a closed-form power (and beamforming in the case of multiple-antenna transmitters) control policy under perfect channel and queue state information so that the objectives (1) are met and (iii) Modification of the obtained algorithms in less demanding information patterns. We derive the analytical model of the system under heavy traffic conditions in Sections 2 and 3, extending the results of [20] and [22]. The main issue in our case is that the transmitting power of each base station affects the rates of all other wireless links, therefore the queue length processes are coupled. Then, once we obtain the Stochastic Differential Equation (SDE) that models the evolution of queues, we apply a special form of linear control to the reserve power to actually decouple the evolution of queues and meet the QoS requirement in Section 4. In that Section we also discuss an implementation of the algorithm in more decentralized settings, where channel states are not known and the case where queue length information becomes available with delays. All the analysis till there is done for continuous time and an asymptotic system as a parameter $n$ goes to infinity; in Section 5 we examine the effect of operating in timeslots and how the parameter $n$ can be chosen in a practical system to actually implement the power control policies. The performance of the control algorithms is then illustrated via simulation of a simple system in Section 6. In this section we also illustrate via simulations the effect of delayed queue state information. Finally, Section 7 concludes the paper.

\section{Notations}

Throughout this paper, bold uppercase letters are used to denote matrices, while bold lowercase letters denote column vectors. Non-boldface letters denote scalars. Therefore, $\mathrm{x}$ will denote the column vector with elements $x_{k}$ and $\|\mathbf{x}\|$ its Eucledean norm. Superscripts $T$ and $H$ over a matrix or vector denotes its transpose and its hermitian respectively, superscript $(n)$ denotes the $\mathrm{n}$-th element of a sequence and superscript \# denotes the pseudoinverse of a matrix, i.e. $\mathbf{A}^{\#}=\mathbf{A}^{H}\left(\mathbf{A A}^{H}\right)^{-1}$. Also, $I_{\{A\}}$ denotes the indicator function, $[a]^{+}$denotes $\max (a, 0), \operatorname{erfc}(x)$ the complementary error function, i.e. $\operatorname{erfc}(x)=\frac{2}{\sqrt{\pi}} \int_{x}^{+\infty} e^{-t^{2}} d t, \operatorname{erfc}^{-1}(x)$ the corresponding inverse function. Finally, $\stackrel{w}{\longrightarrow}$ denotes weak convergence.

\section{System Model and Heavy Traffic Con- DITIONS}

We consider a system of $K$ transmitters each with $L$ antennas serving one receiver and using bandwidth $W$. For notational simplicity, we index the transmitters and receivers so that transmitter $k$ serves user $k$. Let then $g_{i j}(t)$ denote the power gain of the channel between transmitter $j$ and user $i$ at time $t$. Each of these channel gains is assumed to evolve independently of the others as an ergodic finite state Markov chain (for the case of single antenna this model is widely used for fading wireless channels [24]). Under this assumption, the matrix $\mathbf{H}(t)=\left[\mathbf{h}_{i j}(t)\right]$ of all channel gains at time $t$ will also evolve as an ergodic finite state Markov chain with, say, $M_{H}$ possible states and let us index the states as $S_{H}=\left\{1, \ldots, M_{H}\right\}$. We shall denote the event that the channel gains are in the $m$-th state as $\mathbf{H}(t)=\mathbf{H}_{m}$.

The corresponding ergodic probability distribution for each state will then be denoted as $\pi_{m}$, and let $\mathbb{E}_{\pi}\{\}$ denote the expectation over this probability distribution. In our setting no transmitter cooperation, e.g. in the form of joint transmission or space-time coding, is assumed. Therefore, at receiver $k$, the received signals from a transmitter other than $k$ are regarded as interference. In addition, each transmitter will perform a power/beamforming allocation at each time slots. Notice that time sharing and scheduling are not considered in this paper since they require the existence of a central entity that assign the users to time slots. Existence of such entity is not possible in our context 
since we are examining a system with distinct transmitters, each of which communicating only with its own receiver. The interference is treated as Gaussian noise, thus when transmitter $k$ uses beamformer $\mathbf{v}_{k}$, the rate $r_{k}\left(\mathbf{v}_{1}(t), \ldots, \mathbf{v}_{K}(t), \mathbf{G}(t)\right)$ over the corresponding link will be assumed as the Shannon rate, that is [25]

$r_{k}\left(\mathbf{v}_{1}, \ldots, \mathbf{v}_{K}, \mathbf{H}\right)=W \log _{2}\left(1+\frac{\left|\mathbf{v}_{k}^{H}(t) \mathbf{h}_{k k}\right|^{2}}{\sigma^{2}+\sum_{i \neq k}\left|\mathbf{v}_{i}^{H}(t) \mathbf{h}_{i k}\right|^{2}}\right)$.

In the above, $W$ is the bandwidth and $\sigma^{2}$ is the noise variance. The power of transmitter $k$ is given by $p_{k}(t)=\mathbf{v}_{k}^{H}(t) \mathbf{v}_{k}(t)$. In the case of transmitter having single antenna, each channel can be characterized only by its power gain, $g_{k i}(t)$ and the transmitted power is controlled directly. Thus, the received power in the channel from transmitter $k$ to receiver $i$ is $p_{k}(t) g_{k i}(t)$. The rate $r_{k}(\mathbf{p}(t), \mathbf{G}(t))$ is given by $r_{k}(\mathbf{p}(t), \mathbf{G}(t))=$ $W \log _{2}\left(1+\frac{p_{k}(t) g_{k k}(t)}{\sigma^{2}+\sum_{i \neq k} p_{i}(t) g_{i k}(t)}\right)$. For each queue we suppose that the instantaneous arrivals $a_{k}(t)$ at time $t$ are i.i.d., with mean $\lambda_{k}$ and (finite) variance $\sigma_{a, k}^{2}$, and are independent of the arrivals at the other queues and the channel process.

In this work the heavy traffic asymptotic modelling will be used. Informally this means that the average transmission rate at each transmitter will be almost equal to the mean rate of the incoming traffic. Formally, as it is fairly standard in the relevant literature [14], a sequence of systems parametrized by $n$ is created and the system in the limit as $n \rightarrow \infty$ is taken and examined (with time and state variables scaled appropriately). More specifically, the interpretation of the parameter $n$ is such that at any time interval $\Delta t$ there are $O(n \Delta t)$ arrivals, thus $n$ can be seen as the order of magnitude of the arrivals and taking the limit as it goes to infinity implies that in the heavy traffic situations there are too many arrivals in the transmitters. Let $a_{k}^{(n)}(t)$ denote the arrival process at transmitter $k$ at the $n$-th system and $\zeta_{k}^{(n)}(t)$ the corresponding inter-arrival times. Then, for every transmitter $k$ we make the following assumptions [22]:

Assumption 1: The inter-arrival intervals satisfy the following:

1) $\left|\zeta_{k}^{(n)}(l)\right|^{2}$ are uniformly integrable.

2) There exist constants $\bar{\zeta}_{k}^{(n)}, \bar{\zeta}_{k}$ and $\sigma_{k}$ such that

$$
\begin{aligned}
& \mathbb{E}\left\{\zeta_{k}^{(n)}(l)\right\}=\bar{\zeta}_{k}^{(n)} \rightarrow \quad \rightarrow \quad \bar{\zeta}_{k} \quad \text { and } \\
& \lim _{n \rightarrow \infty} \mathbb{E}\left\{\left(1-\frac{\zeta_{k}^{(n)}(l)}{\bar{\zeta}_{k}^{(n)}}\right)^{2}\right\}=\sigma_{k}
\end{aligned}
$$

3) The inter-arrival processes are independent of the channel processes.

The above assumption is roughly equivalent to saying that, for our case, $a_{k}^{(n)}(t) \rightarrow a_{k}(t)$, where $a_{k}^{(n)}(t)$ have mean rate $\lambda_{k}^{(n)} \rightarrow \lambda_{k}$ and $\sigma_{a, k}^{(n)} \rightarrow \sigma_{a, k}$. We would like to stress that that $\lambda_{k}$ and $\sigma_{a, k}$ are finite for every user $k$. Also, denote $\mathbf{v}_{k}^{(n)}(t)$ the power beamforming vector at time $t$ for transmitter $k$ of the $n$-th system. Define $\mathbf{v}^{(n)}(t)$ the column vector containing these beamforming vectors.

A difference in our case with respect to conventional heavy traffic models in wireline networks is that the channels are changing, therefore the time scaling must be done based on the rate on which the channels change rather than the arrival rates. Following [20] and [22], we also parametrize the number of channel changes with the integer $n$ and assume that the channels change also fast but at a slower rate than the incoming traffic. Thus, at a time interval $\Delta t$ there will be $O\left(n^{\nu} \Delta t\right)$ channel changes, for a $0<\nu<1$. Now, let $q_{k}(t)$ denote the queue length of transmitter $k$ at time $t$,and $x_{k}^{(n)}(t)$ the scaled version as follows:

$$
x_{k}^{(n)}(t)=\frac{1}{n^{\frac{\nu}{2}}} q_{k}\left(n^{\nu} t\right) .
$$

The heavy traffic condition regarding the arrival and departure rates will be for every $k$ [22]:

$\lim _{n \rightarrow \infty}\left(\lambda_{k}^{(n)}-\mathbb{E}_{\pi}\left\{r_{k}\left(\mathbf{v}^{(n)}(t)\right)\right\}\right) n^{\frac{\nu}{2}}=\theta_{k}<0, \forall k \in\{1, \ldots, K\}$,

The meaning of the constant $\theta_{k}$ in the equation is that this limit is finite. More specifically, the equation implies that the service rates are bigger than the respective arrival rates, so that the system is stable, but the gap between them closes as $O\left(1 / n^{\frac{\nu}{2}}\right)$. As $n \rightarrow \infty$, the gap between the arrival and service rates becomes very small, thus activating the heavy traffic condition In order for (4) to hold, the beamforming vectors appearing inside the limit have to be of the form

$$
\mathbf{v}_{k}^{(n)}(t)=\overline{\mathbf{v}}_{k}\left(\mathbf{H}^{(n)}(t)\right)+\frac{1}{n^{\frac{\nu}{2}}} \mathbf{v}_{k}^{\prime}\left(\mathbf{x}^{(n)}(t), \mathbf{H}^{(n)}(t)\right) .
$$

In the above expression $\bar{v}_{k}\left(\mathbf{H}^{(n)}(t)\right)$ is such that

$$
\lambda_{k}=\mathbb{E}_{\pi}\left\{r_{k}\left(\overline{\mathbf{v}}\left(\mathbf{H}^{(n)}(t)\right)\right)\right\}, \forall k \in\{1, \ldots, K\} .
$$

From the two equations just presented, it follows that the resource allocation policy consists of two parts: One, denoted $\overline{\mathbf{v}}_{a}$, the column vector of all the beamforming vectors of the first part of (5), based only on the channel states so that the average rate equals the average rate of the incoming traffic (therefore in a sense of equilibrium) and another one, modelled by $\mathbf{u}=\left[\mathbf{v}_{1}^{\prime}(t)^{T}, \ldots, \mathbf{v}_{K}^{\prime}(t)^{T}\right]^{T}$ in (5), based on the queue lengths and probably the channel states. Throughout this work we assume that the arrival rates belong to the rate region achieved by beamforming/power control only, and the main objective is finding a small reserve power such that the QoS constraints (1) are satisfied. In other words, when a power control policy following this rule is implemented, the equilibrium power that corresponds to the realization of the channels at this time is computed and a much smaller additional reserve power so that the total rate is very close to the arrival rateis allocated according to a rule based mainly on the queue lengths. Taking also into account that $n \rightarrow \infty$, (5) implies that at each time $t$ the actual beamforming used by each transmitter is varying slightly around the equilibrium according to the queue 
lengths so that the probabilities that the queue length exceeds a threshold are indeed the desired ones. In the topology of interference channels we are examining here, the limiting factor is interference rather than power constraints so we do not consider such constraints. However, note that if there are power constraints such that (6) cannot hold for some users, these queues will be unstable. On the other hand, since the reserve power is very small, a reasonable power restriction would be to add a fraction of the equilibrium power to the peak power for each user.

Examining (6) further, we can see that there are many possible approaches for the equilibrium power allocation. For example, one may to allocate $\overline{\mathbf{v}}(t)$ so that for any possible state of the channels the rate at each link $k$ will be equal to $\lambda_{k}$, or another approach is to solve the problem of minimizing the expectation over the channel states of total power used with the constraint that (6) is satisfied. As a final remark, unlike the networks considered in the classic heavy traffic literature where the service rates are fixed, in our case the service rates are controlled, depending on the power allocation. This means that the system is actually forced to operate in the heavy traffic regime, with beamforming being as described in (5) and (6) for some large enough scaling parameter $n$.

Finally, some comments with respect to notation are in order. The beamforming vectors are all stacked in the column vector $\mathbf{v}(t)$ with $K L$ elements. Therefore, element $i$ of this vectors corresponds to transmitter $k^{\prime}(i)=\lceil i / L\rceil$ and its antenna indexed by $l^{\prime}(i)=i-\left(k^{\prime}(i)-1\right) L$. The same holds for the equilibrium and reserve beamforming vectors.

\section{Convergence to an SDE}

In this section the actual model of the limit system (as $n \rightarrow \infty)$ in heavy traffic will be obtained as a controlled Stochastic Differential Equation. The derivations make use of the weak convergence methods applied in [20] and [22]. We consider the case when the channel coefficients are real numbers. This is done mainly for mathematical convenience. A way to use the results and methods of this subsection for this more realistic model (with complex channel coefficients) is to examine the power control problem separately for the sine and cosine parts of the signal; then the problem is reduced to two subproblems with real channel coefficients each, and each subproblem is solved with the same way presented in the paper. Our main result follows :

Theorem 2: Consider $K$ interfering links with $L$ antennas at each transmitter. As $n \rightarrow \infty$ the vector-valued process of the scaled queue lengths of (3) is given as,

$$
\mathbf{x}(t)=\mathbf{x}(0)-\int_{0}^{t} \mathbf{f}(\mathbf{u}(s)) d s+\mathbf{\Sigma} \mathbf{w}(t)+\mathbf{z}(t) .
$$

In the above, $\mathbf{w}(t)$ is a vector of $K$ independent standard
Wiener processes, $\mathbf{f}$ is the vector of the functions

$$
f_{k}(\mathbf{u}(t))=\sum_{m=1}^{M_{H}} \pi_{m} \sum_{j=1}^{L K} a_{k, j}\left(\mathbf{H}_{m}\right) u_{j}(t)
$$

where $a_{i, j}\left(\mathbf{H}_{m}\right)=\frac{\partial r_{i}\left(\overline{\mathbf{v}}_{a}\left(\mathbf{H}_{m}\right)\right)}{\partial v_{j}}$. The matrix $\boldsymbol{\Sigma}=\left[\sigma_{i j}\right]$ satisfies

$$
\boldsymbol{\Sigma} \boldsymbol{\Sigma}^{T}=\boldsymbol{\Sigma}_{a} \boldsymbol{\Sigma}_{a}^{T}+\boldsymbol{\Sigma}_{d} \boldsymbol{\Sigma}_{d}^{T}
$$

with $\boldsymbol{\Sigma}_{a}=\operatorname{diag}\left(\sigma_{a, k}\right)$ while the elements of the covariance matrix $\boldsymbol{\Sigma}_{d} \boldsymbol{\Sigma}_{d}^{T}=\left[s_{i j}\right]$ are given as

$$
s_{i j}=2 \mathbb{E}\left\{\int_{0}^{+\infty} \hat{r}_{i}(0) \hat{r}_{j}(t) d t\right\},
$$

where $\hat{r}_{k}(t)=\left(r_{k}(\overline{\mathbf{v}}(\mathbf{H}(t)))-\lambda_{k}\right)$. Finally, the elements of $\mathbf{z}(t)$ are given as

$z_{k}(t)=\left[-\min _{s \leq t}\left\{x_{k}(0)-\int_{0}^{t} f_{k}(\mathbf{u}(s)) d s+\sum_{j=1}^{K} \sigma_{k j} w_{j}(t)\right\}\right]^{+}$.

Proof: Please refer to the Appendix for the proof.

It is interesting to note that, despite most of the cases discussed in the literature (e.g. [20], [14]), in our case the queue lengths are not directly coupled due to the reflection. Generally, the actual reflection term consists of the above process multiplied from the left by a matrix $\mathbf{R}$, denoted in literature as the reflection matrix. The diagonal elements of the reflection matrix are ones, while the off-diagonal elements represent data being routed for transmission to queues that are empty (in other words, if a queue is empty, then it serves some of the data from other non-empty queues). In our case no sort of such cooperation between the transmitters is assumed, so the reflection matrix is the identity. This property will play an important role in selecting the reserve control policy in Section 4.

From now on we will consider the case with single antenna transmitters in order to simplify the notation. This case follows from Theorem 2 putting $L=1$, and we have only power control.In this case the channel gains are scalars $g_{i j}(t)$ with $\mathbf{G}(t)$ the corresponding channel gain matrix, having $M_{G}$ possible states. Note that this model can also accommodate the case where the beamforming direction is fixed at the direction of the equilibrium vector and the reserve part only changes its magnitude.

An interesting thing to note is that we can further simplify the model by considering that the reserve power depends only on the queue lengths. This is reasonable because we can argue that the goal of the reserve power is to regulate the queue lengths while the equilibrium allocation takes care of the channels. In this case we have the following result:

Corollary 3: Consider a reserve control policy that does not depend on the channel states. Then the asymptotic model reduces to

$$
d \mathbf{x}(t)=\mathbf{B u}(\mathbf{x}(t)) d t+\mathbf{\Sigma} d \mathbf{w}(t)+d \mathbf{z}(t)
$$


Proof: In differential form (with the differentials being in the sense of Ito calculus) we have

$$
d \mathbf{x}(t)=-\mathbf{f}(\mathbf{u}(t)) d t+\mathbf{\Sigma} d \mathbf{w}(t)+d \mathbf{z}(t)
$$

with an initial condition $\mathbf{x}(0)=\mathbf{x}_{0}$.

If $\mathbf{u}(t)$ does not depend on the channel state, we can change the order of the sums in (8), so $f_{i}(\mathbf{u}(t))=\sum_{m=1}^{M_{G}} \pi_{m} \sum_{j=1}^{K} a_{i, j}\left(\mathbf{G}_{m}\right) u_{j}(\mathbf{x}(t))=$ $\sum_{j=1}^{K} u_{j}(\mathbf{x}(t)) \sum_{m=1}^{M_{G}} \pi_{m} a_{i, j}\left(\mathbf{G}_{m}\right)$ thus, defining

$$
b_{i j}=-\sum_{m=1}^{M_{G}} a_{i, j}\left(\mathbf{G}_{m}\right) \pi_{m},
$$

we get $f_{i}(\mathbf{u}(t))=-\sum_{j=1}^{K} b_{i j} u_{j}(\mathbf{x}(t))$. Defining the matrix $\mathbf{B}=\left[b_{i j}\right]$, we can write this relation in vector form as $\mathbf{f}(\mathbf{u}(t))=\mathbf{B u}(\mathbf{x}(t))$. This implies that (13) takes the form (12), essentially completing the proof. Moreover, the elements of the matrix $\mathbf{B}$, from (14) are

$$
\begin{gathered}
b_{i i}=-\sum_{m=1}^{M_{G}} \pi_{m} W \ln (2) \frac{g_{i i}\left(\mathbf{G}_{m}\right)}{\sum_{k=1}^{K} g_{k i}\left(\mathbf{G}_{m}\right) \bar{p}_{k}\left(\mathbf{G}_{m}\right)+\sigma^{2}} \\
b_{i j}=\sum_{m=1}^{M_{G}} \pi_{m} W \ln (2) \frac{g_{i i}\left(\mathbf{G}_{m}\right)}{\sigma^{2}+\sum_{k=1}^{K} g_{k i}\left(\mathbf{G}_{m}\right) \bar{p}_{k}\left(\mathbf{G}_{m}\right)} \times \\
\frac{g_{j i}\left(\mathbf{G}_{m}\right) \bar{p}_{i}\left(\mathbf{G}_{m}\right)}{\left(\sigma^{2}+\sum_{k \neq i} g_{k i}\left(\mathbf{G}_{m}\right) \bar{p}_{k}\left(\mathbf{G}_{m}\right)\right)}, i \neq j
\end{gathered}
$$

Note that in this case, the total transmission power takes still into account both the CSI, through the equilibrium part, and the queue states, through the reserve part. For the multiple antenna case the model will be similar, just with $B$ being a $K$-by- $K L$ matrix.

As a final remark for this Section, let us point out that the whole procedure of rescaling time and queue lengths, taking the limit as this scaling factor goes to infinity and using central limit theorems implies that the Stochastic Differential Equation (13) is an averaged model of the system over the random environment and traffic, where statistics up to second order are used.

\section{A Control Policy}

\subsection{Defining equilibrium and reserve control poli- cies with perfect information}

As it can be seen by (5) the resource allocation policy consists of two parts: determining the equilibrium power allocation according to the state of the channels and then determining the reserve power allocation according to the channels and queue lengths in general. In this work, we will set the equilibrium power allocation such that the rates at each possible state of the channels are equal to the mean rates of the incoming traffic. This is equivalent to the problem of maintaining a constant SINR $\bar{\gamma}_{k}$ for each user $k$ for each channel state such that $\lambda_{k}=W \log _{2}\left(1+\bar{\gamma}_{k}\right)$. Therefore, the equilibrium power allocation policy is obtained by solving the following system of linear equations for each $\mathbf{G}_{m}$ and $\forall k \in\{1, \ldots, K\}:$

$$
\frac{1}{\bar{\gamma}_{k}} g_{k k}\left(\mathbf{G}_{m}\right) \bar{p}_{k}\left(\mathbf{G}_{m}\right)-\sum_{i \neq k} g_{i k}\left(\mathbf{G}_{m}\right) \bar{p}_{i}\left(\mathbf{G}_{m}\right)=\sigma^{2}
$$

Here we assume that the channels and incoming traffic characteristics are such that (15) are feasible. If not, then we may still be able to find a suitable equilibrium power allocation; however the general problem in this case is very difficult. Also, methods using time-sharing and scheduling would need the existence of a central authority to schedule transmissions, which is not assumed in this paper. In addition other methods would not be amendable to distributed implementation, as described in the next subsection. In the case of multiple antennas at the transmitters, the problem of finding an equilibrium beamformer so that the corresponding rate is $\lambda_{k}$ for each link can be solved by the algorithm presented in [7].

The reserve power allocation will depend only on the queue lengths, thus the queue dynamics are governed by a stochastic differential equation of the form (12). Note that because the equilibrium allocation given by (15) is such that the corresponding rates are the same for each channel state, there is no randomness in the equilibrium rates therefore there must be $\Sigma_{d}=\mathbf{0}$. In order to find this reserve power allocation policy we will initially work with the asymptotic model (as $n \rightarrow \infty$ ) and impose no constraints on $u_{k}(\mathbf{x}(t))$. All these having been said, we have the following;

Proposition 4: With the equilibrium allocation given as a solution to (15), the overflow requirements for the asymptotic system can be satisfied by the following policy:

$$
\mathbf{u}(\mathbf{x}(t))=\mathbf{B}^{-1} \mathbf{C x}(t)
$$

with $\mathbf{C}=\operatorname{diag}\left(-\left|c_{k}\right|\right)$ and $\left|c_{k}\right|=\frac{1}{2}\left(\frac{\sigma_{a, k}}{x_{k}^{t h r}} \operatorname{erfc} c^{-1}\left(\delta_{k}\right)\right)^{2}$.In the above, $x_{k}^{t h r}$ is the corresponding threshold in the asymptotic regime.

Proof: Let us first start by establishing the probabilistic framework of the stochastic differential equation in (12). Let $\left(\Omega, \mathcal{F},\left\{\mathcal{F}_{t}\right\}_{t \geq 0}, \mathcal{P}\right)$ be a complete probability space satisfying the usual hypotheses, i.e., $\mathcal{F}_{o}$ contains all the $\mathcal{P}$-null sets of $\mathcal{F}$ and $\left\{\mathcal{F}_{t}\right\}_{t \geq 0}$ is a right continuous filtration of $\sigma$-algebras. The Wiener Process $\mathbf{w}(t)=$ $\left(w_{1}(t), \ldots, w_{K}(t)\right)_{t>0}^{T}$ is $\left\{\mathcal{F}_{t}\right\}_{t \geq 0}$-adapted with stationary and independent increments. The Wiener process is also independent of the initial state $\mathbf{x}_{0}$ which is an $\mathcal{F}_{o^{-}}$ measurable random variable with finite second moment. The reflection process $z(t)=\left(z_{1}(t) \ldots z_{K}(t)\right)_{t \geq 0}^{T}$ is a continuous non-decreasing $\left\{\mathcal{F}_{t}\right\}_{t \geq 0}$-adapted $\mathbb{R}_{+}^{K}$ valued process and each $z_{k}(t)$ increase only when $x_{k}(t)=0$. We define our control policy in the class of Markov feedback control (i.e. the control depends on $\mathbf{x}(t)$ ). It is well known that the existence of Markov control is related to the existence of solution for the corresponding SDE. First we will examine the effects of the proposed equilibrium policy 
given by solving (15). Since the corresponding rates are equal to each channel state, there is no randomness in the equilibrium rates therefore the diffusion matrix is now just $\Sigma=\Sigma_{a}$. Taking that into account and applying the proposed controller, the states are decoupled as

$$
d x_{k}(t)=-\left|c_{k}\right| x_{k}(t) d t+\sigma_{a, k} d w_{k}(t)+d z_{k}(t) .
$$

Our control policy makes the evolution of the queues decoupled which is very useful to ensure the existence of a solution to the SDE. Since the states are decoupled and using the reflection direction in (11), we can show that the controlled process $\mathbf{x}(t)$ is positive recurrent. Using the main result of [26], the controlled process in (17) has a unique invariant probability measure which is absolutely continuous with respect to the Lebesgue measure, i.e. has a density that can be obtained using the Fokker-Planck equation as $\phi\left(x_{k}\right)=\sqrt{\frac{\left|c_{k}\right|}{\pi \sigma_{a, k}^{2}}} e^{-\left|c_{k}\right| x_{k}^{2} / \sigma_{a, k}^{2}}$. According to the ergodic properties of recurrent diffusion processes, we can use the above density of the invariant measure to compute the overflow of the controlled stochastic process $x(t)$

$$
\operatorname{Pr}\left\{x_{k}(t)>x_{k}^{t h r}\right\}=\operatorname{erfc}\left(\frac{x_{k}^{t h r} \sqrt{2\left|c_{k}\right|}}{\sigma_{a, k}}\right) .
$$

Replacing the overflow probability with its desired value and solving (18) completes the proof.

Note that the control policy regarding the reserve power is a closed form expression of the queue lengths. Given that the equilibrium power allocation is precomputed and under the assumption of complete queue and CSI knowledge this implies that at each time the control policy can be implemented in one shot instead of having an iterative algorithm (as it is done e.g. in [11]). In addition, let us point out that the above results are in the steady state, i.e. in the sense that the process is running for an infinite time horizon. Moreover, looking at (8) we can observe that the drift term of the Stochastic Differential Equation of the limit model is in fact an expectation over the ergodic distribution of the channel gains process, therefore the overflow probabilities calculated here are approximations - but quite accurate ones for many cases as we will see in the simulations section.

Finally, let us point out that the form of the power control policy is heuristic such that the overflow constraints (1) are satisfied. Restricting the reserve power to depend only on the queue lengths and be linear with respect to them simplified the problem quite substantially. In principle it is possible that another choice of this control may still lead to the desired result with respect to the individual QoS constraints while minimizing some cost function. However, the resulting optimal control problem is intractable to solve analytically, and a solution satisfying the constraints (1) is not even guaranteed to exist. Also, as we will see in the simulations, the reserve power is much smaller than the equilibrium power so the heuristic performs well in terms of energy efficiency.
For the multiple antenna case the procedure is the same, with the only difference that in the control policy there needs to be the pseudoinverse of the corresponding matrix $\mathbf{B}$.

\subsection{Control policy with local SINR feedback}

So far, we have assumed that at each time slot there is full knowledge of the realizations of all the channels and queue lengths and the calculation of the transmission powers was done based on that knowledge. However, this assumption is unrealistic in practice. In this subsection we will examine the case where each receiver can send SINR feedback to its corresponding transmitter but other than that no information on the channels is available. Taking into account that the equilibrium power allocation is such that the corresponding rate is constant, we can use the algorithm proposed in [8] to find the equilibrium power allocation without the need for knowledge of all channel realizations. More specifically, for a system adjusting the power in discrete time, in the beginning of each time slot we can dedicate a time $\tau$ where the transmitters find the equilibrium power. In order to do that,each transmitter $k$ requires only the SINR feedback from its corresponding receiver and runs the following iterative process

$$
p_{k}(i+1)=\frac{\bar{\gamma}_{k}}{\gamma_{k}(i)} p_{k}(i),
$$

where $i$ denotes here the iteration of the algorithm, $\bar{\gamma}_{k}$ the target SINR of user $k$ and $\gamma_{k}(i)$ the SINR at this user after the power update of iteration $i$. It is shown in [8], [6] that, for a given but unknown realization of the channels, this algorithm indeed converges to the equilibrium values corresponding to these channels and moreover this convergence is very fast (the rate of convergence is exponential). This analysis implies that the only real time information truly needed to implement the proposed power control policy are the queue lengths at the beginning of each time slot (they are still required to compute the reserve power). Also, the statistics of the channels and incoming traffic processes are still needed in order to find the parameters in the SDE that models the evolution of the queue lengths.

As a result of the training period, the actual data transfer is taking place for a duration of $\left(T_{s}-\tau\right)$ in a time slot of duration $T_{s}$, instead of its whole duration. A way to take this into account is to adjust the bandwidth in (2) appropriately, i.e. if $W^{\prime}$ is the physical available bandwidth, the rates and all parameters in the models are calculated using bandwidth

$$
W=\frac{T_{s}-\tau}{T_{s}} W^{\prime} .
$$

In the above, $\tau$ is assumed fixed and taken such that it is enough for the algorithm given by (19) to converge.

For the case of multiple antenna transmitters, each transmitter can acquire the channel realization of its respective receiver. See [7] for an algorithm requiring 
little information exchange between transmitters and [27] for a decentralized algorithm to achieve the desired equilibrium rates.

\subsection{Control policy in the case of delayed queue state information}

Another major issue for the practical implementation of our control policy is the requirement that each transmitter is aware of all the queue lengths instantaneously. A more realistic assumption would be that each transmitter knows its own instantaneous queue state and has access to delayed information about the queues of the others. For example this can correspond to a Small Cell Network setting where the base stations exchange information using a backhaul of limited capacity. We will assume though that each transmitter knows the statistics of the arrivals to the other transmitters. To simplify the analysis, we will assume that the delay in sharing the queue length information is the same for any pair of transmitters, i.e. at time $t$, transmitter $i$ has access to the queue length of transmitter $j$ at time $t-\tau_{d}$.

Let us define the observation of the queue length of transmitter $i$ at time $t$ as $\hat{x}_{i}(t)=x_{i}\left(t-\tau_{d}\right)$ and the vector of the observations of the queue states at transmitter $i$ as $\hat{\mathbf{x}}_{k}(t)$. Then the following holds

Proposition 5: In the case where the queue state information is exchanged with delay $\tau_{d}$ and the control of Section 4 is applied, the asymptotic model evolves as

$d \mathbf{x}(t)=\mathbf{C x}(t) d t+\mathbf{B D}_{L}(\tilde{\mathbf{x}}(t)-\mathbf{x}(t)) d t+\mathbf{\Sigma} d \mathbf{w}(t)+d \mathbf{z}(t)$.

The matrix $\mathbf{D}_{L}$ is given as the matrix $\mathbf{L}=\mathbf{B}^{-1} \mathbf{C}$ with its diagonal elements replaced by zeros, $\tilde{\mathbf{x}}(t)=x_{k}\left(t-\tau_{d}\right)$.

Proof: Denoting $\mathbf{L}=\left[l_{i j}\right]$, the control using the delayed observations is given by $u_{k}(t)=l_{k k} x_{k}(t)+$ $\sum_{i \neq k} l_{k i} x_{i}\left(t-\tau_{d}\right)$. In a vector form, and using $\mathbf{L}=$ $\operatorname{diag}\left\{l_{k k}\right\}+\mathbf{D}_{L}$ we get $\mathbf{u}(t)=\operatorname{diag}\left\{l_{k k}\right\} \mathbf{x}(t)+\mathbf{D}_{L} \tilde{\mathbf{x}}(t)=$ $\mathbf{L x}(t)+\mathbf{D}_{L}(\tilde{\mathbf{x}}(t)-\mathbf{x}(t))$. Replacing in (12) and taking into account that $\mathbf{L}=\mathbf{B}^{-1} \mathbf{C}$ we get the stated result.

An observation that can be made is that in the case of delayed queue length information, a term proportional to the difference between the vector of observations (delayed versions) of the queues and the vector of the actual queue lengths $\mathbf{e}(t)=\tilde{\mathbf{x}}(t)-\mathbf{x}(t)$ is added to the original evolution of the queue lengths.

In order to analyze the impact of the delay, consider the case where this delay in information sharing is infinite. In this case, no information about the queue lengths is in fact shared among the transmitters so their estimations cannot be updated. Thus, the estimation vector in (21) will be a constant $\tilde{\mathbf{x}}$ (which can correspond for example to an initial estimation). In this case, replacing $\tilde{\mathbf{x}}(t)=\tilde{\mathbf{x}}$ in (21), we get that the state evolves according to the equation $d \mathbf{x}(t)=\left(\mathbf{B D}_{L} \tilde{\mathbf{x}}-\mathbf{A x}(t)\right) d t+\boldsymbol{\Sigma} d \mathbf{w}(t)+d \mathbf{z}(t)$, where $\mathbf{A}=\left[a_{i j}\right]=\mathbf{C}-\mathbf{B D}_{L}$. This case is the worst case scenario, as each transmitter gets no information at all about the evolution of the queue lengths of the others, and can be used to find the upper bound of the overflow probabilities for any estimation scheme (as the general case with a finite delay is very difficult to be analyzed). Denote $\phi(t, \mathbf{x})$ as the density of the joint probability distribution of the queue lengths at time $t$. Then, from the theory of Stochastic Differential Equations, it follows the corresponding Fokker Planck equation:

$$
\frac{\partial}{\partial t} \phi(t, \mathbf{x})=-\sum_{i=1}^{K} a_{i i} \phi(t, \mathbf{x})+\sum_{i=1}^{K} \frac{\sigma_{a, i}^{2}}{2} \frac{\partial^{2}}{\partial x_{i}^{2}} \phi(t, \mathbf{x})
$$

with the appropriate initial conditions for $t=0$ and at the reflecting barrier (i.e. at all points $\mathrm{x}$ with at least one element zero) [28]. This equation can be solved only numerically and the invariant measure as $t \rightarrow \infty$ can be taken. Then, using the marginal distributions we can obtain numerical results for the queue overflow probabilities.

A way to use this is to adjust the threshold appropriately: If the overflow probability at some transmitters are larger than desired, we can decrease the corresponding thresholds and derive the control policy for these decreased thresholds. The overflow probability then for the initial (bigger) thresholds will be smaller. This procedure can be repeated until we get acceptable overflow probabilities for the initial desired thresholds.

For the case where the delay in information sharing is not infinite, a simple heuristic approach is that each transmitter calculates the transmission power with the queue length vector being replaced with the vector of the most recent information about the queue states this transmitter has. While there are no information yet about the queue at a transmitter $i$, the transmitter $k$ uses the standard deviation of the incoming traffic at $i$ properly modified taking into account the time slot duration, i.e. $\sqrt{T_{s} \sigma_{a, i}^{2}}$, as an estimation of the queue length. This scheme will intuitively perform better than the worst case scenario (with no queue length information of other transmitters) described earlier, however it is very difficult to analyze.

\section{Discrete TIME IMPLEMENTATION ISSUES}

So far we worked on continuous time models whereas in the real communications system time is slotted and power allocation decisions are taken into discrete time instances. More specifically, let the duration of each timeslot of the unscaled system be $T_{s}$; this implies that at the $n$-th system of the sequence the timeslot duration will be $T_{s}^{(n)}=T_{s} n^{-\nu}$. Then, in the general case where the equilibrium allocation is such that $\Sigma_{\mathrm{d}} \neq \mathbf{0}$, the departure process converges to a Wiener process with covariance matrix given as $s_{i j}^{\prime}=T_{s} \mathbb{E}_{\pi}\left\{\hat{r}_{i}(0) \hat{r}_{j}(0)\right\}+$ $2 T_{s} \mathbb{E}\left\{\sum_{l=1}^{+\infty} \hat{r}_{i}(0) \hat{r}_{j}(l)\right\}$ (see e.g. [20]). The expectations are taken again with respect to the distribution of the channels and $\hat{r}_{j}(l)$ are the discrete time versions of the corresponding quantities given by (57) in the Appendix. 
The analysis and explanation of the above equation is the same as in continuous time.

The derivation of the control policy was concerned with an asymptotic system model. However, it has to be modified in order to operate on a real system, so the results obtained for the asymptotic case have to be converted into results for the unscaled system. This will be done using (3) and (5), where this scaling parameter $n$ is now a "big enough" finite number. By the definition that at a time interval $\delta t$ there are $O(n \delta t)$ arrivals, we can argue that in practical cases $n$ can be the order of magnitude of the average bit rates of the incoming traffic in the system. Also, as far as the exponent $\nu$ is concerned, once $n$ is fixed it can be obtained using the fact that during period with duration equal to the coherence time, $T_{c o h}$, there must be only one channel change, thus $n^{\nu} T_{\text {coh }}=1$.

In the specific control policy presented in the previous section, the equilibrium rates are the same with the mean rates so the covariance matrix of the limit Wiener process that corresponds to the departure process will be zero regardless of the fact that the system operates in time slots. Note however that we have the assumption that the channel change slower than the arrival process, so we can assume that the channel stays the same within a timeslot. Based on the previous analysis, at the "real" system where the queue lengths are $\mathbf{q}(l)$ at the beginning of timeslot $l$ and the channel gain matrix is at state $m$, the power allocated for the duration of this timeslot will become (applying time slotting and unscaling in the results of Section 4): $\mathbf{p}(l)=\overline{\mathbf{p}}(\mathbf{G}(l))+\mathbf{B}^{-1} \mathbf{C}^{\prime} \mathbf{q}(l)$ where $\mathbf{C}^{\prime}=\operatorname{diag}\left(\left|c_{k}^{\prime}\right|\right)$ is obtained by Proposition 4 replacing the scaled queue length threshold with its real unscaled value, $q_{k}^{t h r}$. Note also that since in (18) both quantities inside the probability are scaled ones, this is also the probability of the unscaled queue lengths exceeding their respective unscaled thresholds.

From section 4 it is implied that it is possible to find a control policy of the type discussed for any values of the queue thresholds and overflow probabilities, which intuitively not the case. In fact, some limitations are given by the following proposition:

Proposition 6: Consider a time slotted system with slots of length $T_{s}$ for data transmission that operates updating the power allocation at the start of each time slot as described in this Section. Then, for each transmitter $k$, the achievable queue length thresholds to be exceeded with probability $\delta_{k}$ are bounded as

$$
q_{k}^{t h r} \geq \frac{\sigma_{a, k}}{2} \sqrt{T_{s}} \operatorname{erfc}^{-1}\left(\delta_{k}\right)
$$

Proof: The discrete time dynamics of the real system can be approximated from (17) taking into account the form of the expression for the total power as $\left(n_{k}(l)\right.$ is the discrete time AWGN process with unitary variance): $q_{k}(l+1)=\left[\left(1-\left|c_{k}^{\prime}\right| T_{s}\right) q_{k}(l)+T_{s} \sigma_{a, k} n_{k}(l)\right]^{+}$. For the above difference equation not to diverge, there must be $|1-| c_{k}^{\prime}\left|T_{s}\right| \leq 1$, therefore $\left|c_{k}^{\prime}\right| \leq \frac{2}{T_{s}}$. Replacing $\left|c_{k}^{\prime}\right|$ using the analysis in section 4, we get the stated relation.

This threshold bound is an approximation and it illustrates the effect of the discrete time operation in an actual system. The same results hold for the multiple antenna case. The results in this Section are based on heuristics and are approximate. Obtaining exact results for the initial discrete time system is, as far as we are aware of, generally an open issue in the area of analysing and designing systems via traffic approximations. However, these approximate results provide useful insights.

\section{Simulation Results}

In order to illustrate the results of the power control method presented in sections 4 and 5, let us consider a simple scenario with 3 interfering transmitter - receiver pairs, using a the same spectrum with bandwidth $5 M H z$. For simplicity, consider that each channel gain has only two possible values. Also, the arrivals at each transmitter were set as Poisson processes with mean rates $1,1.5$ and $2 \mathrm{Mbps}$. The overflow thresholds are $500,750,1000$ bits at each transmitter respectively and the overflow probability is 0.01 for all transmitters. The coherence time of the channels is set to $20 \mathrm{~ms}$, corresponding to slow fading channels like in indoor and low mobility environments. The time slot duration is $2 \mathrm{~ms}$, motivated by the shortest scheduling interval in HSDPA, thus the channel stays the same for 10 consecutive power configurations. The noise variance was set to 0.01 at each receiver.

Based on the above settings and the analysis in Section 5 , for the simulated system a reasonable value of $n$ can be $n=1000000$, the order of magnitude of the incoming traffic at all users, and $\nu$ can be such that $n^{\nu} T_{c o h}=1$, thus $\nu=0.283$.

The maximum equilibrium power is $0.0083 \mathrm{~W}$, while all the equilibrium powers are in the order of magnitude of $m W$. The expected values of the equilibrium powers over the ergodic distribution of the channel gains matrix are found to be $0.0043 \mathrm{~W}, 0.0062 \mathrm{~W}, 0.0081 \mathrm{~W}$ for transmitters 1, 2 and 3 respectively. The average reserve powers used where found (by simulations) to be around $0.7 \times 10^{-5} \mathrm{~W}, 0.7 \times 10^{-5} \mathrm{~W}, 0.8 \times 10^{-5} \mathrm{~W}$.

Our proposed method is compared with the policy where all powers take their equilibrium values according to the channel states, and the following heuristic power allocation strategies: One with the power at each transmitter being constant for every queue length and channel state and equal to the expectation of the equilibrium power over the ergodic distribution of the channel gain matrix plus the average reserve power presented above and one with the power being the one given adding the equilibrium allocation. In the presentation of the results, the former will be denoted as "Static power allocation" and the latter as "Channel-aware power allocation". These configurations were made to ensure that the amount of power available in the heuristic schemes is approximately the same as the power used in 
our proposed method, thus making a fair comparison. We will show the results concerning the queue of one transmitter, the one for Link 1, as all the results are very similar.

The performance of the aforementioned power allocation policies for each queue are shown in Figure 2. The static power allocation performs aite bad as it does not take into account at all the channel states and the queue lengths. As far as the equilibrium power allocation is concerned, the bad performance is explained because the traffic and the queue lengths are not taken into account. Analyzing this policy further, (12) implies that putting the vector of the reserve powers $\mathbf{u}$ equal to zero, the queue lengths behave like reflecting Wiener processes. This is illustrated in our simulation setting in Figure 3. In Figure 2 it is clearly illustrated that assigning slightly more power in the equilibrium power for each channel state, as it is done in the channelaware power allocation scheme defined earlier, leads to a much better performance, even if this extra power is really small. This was also expected as an increase in all the powers leads to an increase in the average rates. The equilibrium power allocation is exactly the point where the mean arrival rates equal the mean service rates thus even a small increase of the mean service rates is enough to stabilize the system. Achieving a better performance than the static power allocation case was also expected due to the transmission powers adapting to the channel conditions. Finally, we can observe that our proposed method of power allocation does even better, illustrating the additional advantage of taking the queue lengths into account when allocating the reserve power. Moreover, the overflow ratio is very close to the desired one, which implies the validity of the asymptotic model in a practical system operating under heavy load.

In Figure 3 we can also see the evolution of the queue length over time for a simulation run. We can observe that the queue length under the proposed power control method behaves in a much more controlled manner compared to the equilibrium power allocation and is below its respective threshold for most of the time. Finally, Figure 4 depicts the power allocated using our proposed policy and the equilibrium power allocation for the transmitter of Link 1 for the first 100 instances of a simulation run. We can see that the total allocated power varies slightly around the equilibrium power level in each channel state, which clearly demonstrates the effect of allocating some extra power according to the queue lengths. We can see that this variation around the equilibrium power is indeed very small, thus confirming the assumption of very small reserve power.

As a next step, a simple system with transmitters with multiple antennas is simulated in order to verify the theoretical results and illustrate the impact of multiple antennas in the operation of the system. For computational purposes, we consider a network of two links for the cases where the transmitters have one, two and three antennas. The channels from each antenna to each receiver are assumed i.i.d. two state Markov chains and the incoming traffic processes are again Poisson distributed with mean rates 1 and 2 Mbps for links 1 and 2 respectively. We set the respective thresholds to 500 and 1000 bits and the desired overflow probability to 0.01 . For any given number of antennas, both beamforming control methods (i.e. fixed direction for a given realization of channel states and direction of the reserve vector depending on the queue length) are considered, operating both for the same instances of incoming traffic and channel realizations.

A result from these simulations is that for each simulation run and a given number of antennas (that is the same traffic and channel realizations) the overflow ratios were the same for each beamforming method used. Indeed, from the theoretical analysis it holds that for any beamforming method presented here, the control is such that evolution of the queues follows the same equation (17). Moreover, Figure 5 shows the Cumulative Distribution Function of the overflow probabilities over the simulation runs; that is in for each curve the $y$ axis shows the ratio of simulation runs where the overflow ratio was smaller than the corresponding point of the curve at $x$ axis. We can see that the overflow ratio was indeed very close to the desired one. These results illustrate the validity of the asymptotic approach in a practical system. Regarding the evolution over time, it is similar as in the case with 3 links and one antenna (see Figure 3).

Figure 6 depicts the average total power consumption of the system in each simulation run for each of the cases examined. In the cases of multiple antennas, the average power used was almost the same for the two beamforming methods, so we present just one plot for each number of antennas. These results imply that adding more antennas at the transmitters, under the assumption that the corresponding paths are independent, increases the energy efficiency of the system for the same requirements in terms of buffer overflows.

Finally, we study through simulations the effect of delayed queue state information in our algorithm. For simplicity, all the delays in information sharing between the transmitters are set to be the same. Performance in terms of overflow ratios are given in Fig. 7 as the CDFs over the simulation runs (in the same sense as in Fig. 5). As we can see in this figure, as the delay in information sharing increases the overflow ratios tend to be higher. However, especially for small delays, the differences are still relatively small. Also, even for relatively high delay in information sharing the overflow ratios tend to be not very far from the desired one. Thus we can argue that knowing the incoming traffic statistics at each base station, our proposed scheme seems to be quite robust in cases of delayed information sharing.

\section{Conclusions}

In this paper we have used the heavy traffic approximation in order to model and propose algorithms for 
the power allocation problem for interfering wireless links, which results in an asymptotic but tractable way to analyze the problem and derive some control strategies. The objective was to keep desired overflow probabilities at each queue assuming that the channels change according to an ergodic finite state Markov chain. The allocated power is split into two: a part that is allocated according to the channels and a much smaller part that is allocated according to the backlogs at the queues at each time, for which closed form expression as a function of the queue lengths was derived. The advantage of the algorithm is that it can be implemented in one shot at the beginning of each time slot. This work was also extended for the case when the transmitters have multiple antennas, where beamforming methods were proposed following the same approach. Even though the model derived and used is an approximation, simulation results have shown that for reasonable thresholds and overflow probabilities, direct application of the policy derived from the asymptotic system can give quite accurate results. In addition, simulation results imply that the algorithm is quite robust in the case of delayed queue state information.

\section{APPENDIX \\ ProOF OF THEOREM 2}

In order to prove the convergence of the scaled queue to an SDE, we adopt an approach similar to the one used in [20] [22]. Recall that [20] deals with centralized power control in a multiuser downlink system with orthogonal transmissions among users (no interference) and single antenna system. [22] deals with power control for a point-to-point single antenna and single user wireless channel. Given the form of the beamforming vectors, we can write the rates at the $n$-th system as $r_{k}^{(n)}\left(\mathbf{v}_{a}(t), m\right)=r_{k}\left(\overline{\mathbf{v}}\left(\mathbf{H}_{\mathbf{m}}\right)+\frac{1}{n^{\frac{v}{2}}} \mathbf{u}(\mathbf{x}(t), m)\right)$ and expand it in a Taylor series around $\overline{\mathbf{v}}\left(\mathbf{G}_{\mathbf{m}}\right)$ as $n \rightarrow \infty$. We will then get

$$
\begin{aligned}
r_{k}^{(n)}(t)= & r_{k}\left(\overline{\mathbf{v}}\left(\mathbf{H}_{m}\right)\right)+ \\
& \frac{1}{n^{\frac{\nu}{2}}} \sum_{i=1}^{L K} \frac{\partial}{\partial v_{i}}\left(r_{k}\left(\overline{\mathbf{w}}_{a}\left(\mathbf{H}_{m}\right)\right)\right) u_{i}\left(\mathbf{x}(t), \mathbf{H}_{m}\right)+_{O}\left(n^{-\frac{\nu}{2}}\right)
\end{aligned}
$$

Denoting $r_{k}\left(\overline{\mathbf{w}}\left(\mathbf{H}_{m}\right)\right)=\bar{r}_{k}\left(\mathbf{H}_{m}\right)$ (as in fact these rates depend only on the channel state), we can write the rate (24) as

$$
r_{k}^{(n)}(t)=\bar{r}_{k}\left(\mathbf{H}_{m}\right)+\frac{1}{n^{\frac{\nu}{2}}} \sum_{i=1}^{L K} a_{k, i}\left(\mathbf{H}_{m}\right) u_{i}\left(\mathbf{x}^{(n)}(t), \mathbf{H}_{m}\right) .
$$

If $d_{k}(l)$ are the amounts of data transmitted from transmitter $k$ at timeslot $l$, we have $q_{k}(l+1)=\left[q_{k}(l)+\right.$ $\left.a_{k}(l)-d_{k}(l)\right]^{+}$. Denoting now $A_{k}(t), D_{k}(t)$ the arrivals and total bits that could have been transmitted (if the queue was always full) up to time $t$ respectively, we get

$$
q_{k}(t)=\left[q_{k}(0)+A_{k}(t)-D_{k}(t)\right]^{+} .
$$

Now let us construct the sequence of systems whose limit will be eventually the heavy traffic model of the system. Recall that $x_{k}^{(n)}(t)=\frac{1}{n^{\nu}} q_{k}\left(n^{\nu} t\right)$ and also let us define the centered around the mean rates versions of the total arrival and data transmission processes as :

$$
\bar{A}_{k}^{(n)}(t)=\frac{1}{n^{\frac{\nu}{2}}} \int_{0}^{n^{\nu} t}\left(a_{k}^{(n)}(s)-\lambda_{k}\right) d s
$$

and

$$
\bar{D}_{k}^{(n)}(t)=\frac{1}{n^{\frac{\nu}{2}}} \int_{0}^{n^{\nu} t}\left(\bar{r}_{k}\left(\mathbf{H}^{(n)}(s)\right)-\lambda_{k}\right) d s
$$

respectively. Also define the scaled amount of data transmitted due to only the reserve allocation up to time $t$ (assuming always full queue) as

$$
\begin{aligned}
& F_{k}^{(n)}(t)= \\
& \left.\frac{1}{n^{\nu}} \int_{0}^{n^{\nu} t} \sum_{m=1}^{M_{H}} I_{\left\{\mathbf{H}^{(n)}(s)=\mathbf{H}_{m}\right\}} \sum_{i=1}^{L K} a_{k, i}\left(\mathbf{H}_{m}\right) u_{i}\left(\mathbf{x}(s), \mathbf{H}_{m}\right)\right) d s .
\end{aligned}
$$

In this case, we can rewrite (26) as

$$
x_{k}^{(n)}(t)=x_{k}^{(n)}(0)-F_{k}^{(n)}(t)-\bar{D}_{k}^{(n)}(t)+\bar{A}_{k}^{(n)}(t)+z_{k}^{(n)}(t) .
$$

In the above, $z_{k}^{(n)}(t)$ are processes to satisfy the physical constraint that each of the scaled queue lengths $x_{k}^{(n)}(t)$ is nonnegative.

We will now, similar to [20, 22], examine the convergence as $n \rightarrow \infty$ of each one of the terms in the above equation separately.

\section{Arrival Process}

Given that $a_{k}^{(n)}(s)$ are i.i.d. in time and that $a_{k}^{(n)}(s) \rightarrow$ $a_{k}(s)$ where $a_{k}(s)$ has finite mean $\lambda_{k}$ and variance $\sigma_{a, k}^{2}$, from an extension of the Functional Central Limit Theorem [11] it holds that as $n \rightarrow \infty, \bar{A}_{k}^{(n)}(t)$ converges weakly to a Wiener process with zero drift and variance $\sigma_{a, k}^{2}$ :

$$
\bar{A}_{k}^{(n)}(t) \stackrel{w}{\longrightarrow} \sigma_{a, k} w_{a, k}(t) .
$$

In the above, $w_{a, k}(t)$ denotes the standard Wiener process.

The above convergence happens for every $k$ and recalling that incoming traffic flows are independent, the vector containing these arrival processes as elements converges weakly as

$$
\mathbf{a}^{(n)}(t) \stackrel{w}{\rightarrow} \boldsymbol{\Sigma}_{a} \mathbf{w}_{a}(t)
$$

where $\boldsymbol{\Sigma}_{a}=\operatorname{diag}\left(\sigma_{a, k}\right)$ and $\mathbf{w}_{a}(t)$ is a vector of independent standard Wiener processes. 


\section{.2 Service Process}

In order to find the limit of the departure process, we will follow a method similar as before. More specifically, we can write (28) as $\bar{D}_{k}^{(n)}(t)=$ $\frac{1}{n^{\frac{\nu}{2}}} \int_{0}^{n^{\nu} t} \sum_{m=1}^{M_{H}} I_{\{\mathbf{H}(s)=m\}} \bar{r}_{k}(m) d s-\lambda_{k} n^{\frac{\nu}{2}} t$. We define now $\tilde{r}_{k}(t)=\sum_{m=1}^{M_{H}} I_{\left\{\mathbf{H}(t)=\mathbf{G}_{m}\right\}} \bar{r}_{k}\left(\mathbf{H}_{m}\right)$; then by the definition of the indicator function the vector $\tilde{\mathbf{r}}(t)$ is a finite state Markov chain evolving as the channels with values $\overline{\mathbf{r}}\left(\mathbf{H}_{m}\right)$ when the channel processes are at state $m$. Denote now also

$$
\hat{\mathbf{r}}(t)=\tilde{\mathbf{r}}(t)-\lambda .
$$

Taking into account the equilibrium power allocation, we have $\lambda_{k}=E\left\{\hat{r}_{k}(t)\right\}$ and following [20] we have that the above converges weakly to a $K$-dimentional Wiener process with covariance matrix $\mathbf{S}_{d}=\left[s_{i j}\right]$ such that

$$
s_{i j}=2 \mathbb{E}\left\{\int_{0}^{+\infty} \hat{r}_{i}(0) \hat{r}_{j}(t) d t\right\} .
$$

By comparison, this process is exactly the multidimentional process that has $\bar{D}_{k}^{(n)}(t)$ as its $k$-th element. So denoting $\boldsymbol{\Sigma}_{d}=\left[\sigma_{i j}\right]$ such that $\boldsymbol{\Sigma}_{d} \boldsymbol{\Sigma}_{d}^{T}=\mathbf{S}_{d}$, we have for each $k$

$$
\bar{D}_{k}^{(n)}(t) \stackrel{w}{\rightarrow} \sum_{j=1}^{K} \sigma_{k j} w_{d, j}(t)
$$

where $w_{d, j}(t)$ independent standard Wiener processes.

It has to be noted here that, unlike the centered arrival processes, the centered transmission processes are not independent in the limit. That was expected because essentially they depend upon the same process (the one that governs the channel gain matrix). The elements of the covariance matrix of the limiting Wiener process are actually integrals of the temporal correlation between the centered processes at the queues and actually depend on the temporal correlation of the Markov chain modelling the channel gains. In the case that channel gains were i.i.d. over time, only the correlation at the same time instance $(t=0)$ would appear. However, as the Markov chain is ergodic, from some time onwards will have reached its invariant distribution and the correlations with the initial time would be zero. Finally, for the special case where the equilibrium allocation is such that $\bar{r}_{k}(m)=\lambda_{k}$ for every $k$ and $m$, the centered departure process is just the zero process.

As far as the reserve power is concerned, in the limit as $n \rightarrow \infty$, applying the Functional Law of Large Numbers we obtain $F_{k}^{(n)}(t) \stackrel{w}{\rightarrow} \int_{0}^{t} \sum_{m=1}^{M_{H}} \pi_{m} \sum_{i=1}^{L K} a_{k, i}\left(\mathbf{H}_{m}\right) u_{i}\left(\mathbf{x}(s), \mathbf{H}_{m}\right) d s$. With $f_{k}(\mathbf{u}(s))$ given as in (8), this implies

$$
F_{k}^{(n)}(t) \stackrel{w}{\rightarrow} \int_{0}^{t} f_{k}(\mathbf{u}(s)) d s .
$$

For completeness, we present the expressions for the coefficients $a_{k, i}\left(\mathbf{H}_{m}\right): \quad a_{k, i}^{\prime}\left(\mathbf{H}_{m}\right)=$ $W \ln (2) \frac{2 h_{k^{\prime}(i) k}^{\left(l^{\prime}(i)\right)}\left(\mathbf{H}_{m}\right)\left|\overline{\mathbf{w}}_{k}^{H}\left(\mathbf{H}_{m}\right) \mathbf{h}_{k^{\prime}(i) k}\left(\mathbf{H}_{m}\right)\right|}{\sigma^{2}+\sum_{j=1}^{K}\left|\overline{\mathbf{w}}_{j}^{H}\left(\mathbf{H}_{m}\right) \mathbf{h}_{j k}\left(\mathbf{H}_{m}\right)\right|^{2}}$, for $k^{\prime}(i)=k$ and $a_{k, i}^{\prime}\left(\mathbf{H}_{m}\right)=-W \ln (2) \frac{2 h_{k^{\prime}(i) k}^{\left(l^{\prime}(i)\right)}\left(\mathbf{H}_{m}\right)\left|\overline{\mathbf{w}}_{k}^{H}\left(\mathbf{H}_{m}\right) \mathbf{h}_{k k}\left(\mathbf{H}_{m}\right)\right|^{2}}{\sigma^{2}+\sum_{j=1}^{K}\left|\overline{\mathbf{w}}_{j}^{H}\left(\mathbf{H}_{m}\right) \mathbf{h}_{j k}\left(\mathbf{H}_{m}\right)\right|^{2}} \times$ $\frac{\left|\overline{\mathbf{w}}_{k^{\prime}(i)}^{H}\left(\mathbf{H}_{m}\right) \mathbf{h}_{k^{\prime}(i) k}\left(\mathbf{H}_{m}\right)\right|}{\sigma^{2}+\sum_{j \neq k}\left|\overline{\mathbf{w}}_{j}^{H}\left(\mathbf{H}_{m}\right) \mathbf{h}_{j k}\left(\mathbf{H}_{m}\right)\right|^{2}}$, for $k^{\prime}(i) \neq k$.

\section{.3 Reflection Process}

In order to complete the model, we have to examine the processes $z_{k}^{(n)}(t)$. As mentioned earlier, $z_{k}^{(n)}(t)$ must be such that the queue lengths remain nonnegative, thus defining what exactly happens at the time instance when the queue of transmitter $k$ is empty (i.e. there are no data to send to its receiver). In our system setting each transmitter serves only its own receiver and the equilibrium power is assigned even if the queue is empty at the power allocation instance. Therefore, when a base station is allocated power when its queue is empty, the power is wasted and the process $z_{k}^{(n)}(t)$ corresponds to just the amount of data that could have been sent during this transmission; this amount has to be subtracted from the result of the other terms in (30):

$$
z_{k}^{(n)}(t)=\frac{1}{n^{\nu / 2}} \int_{0}^{n^{\nu} t}\left(r_{k}^{(n)}(s)-a_{k}^{(n)}(s)\right) I_{\left\{x_{k}^{(n)}(s)=0\right\}} d s .
$$

The above implies that this process increases only when $x_{k}^{(n)}(t)$ hits zero. The reflection process that satisfies these requirements is [21]

$z_{k}^{(n)}(t)=\max \left\{0,-\min _{s \leq t}\left\{x_{k}^{(n)}(0)+A_{k}^{(n)}(s)-D_{k}^{(n)}(s)\right\}\right\}$.

Indeed, note that $\max \left\{0,-\min _{s \leq t}\left\{x_{k}^{(n)}(0)-D_{k}^{(n)}(t)+\right.\right.$ $\left.\left.A_{k}^{(n)}(t)\right\}\right\} \geq-\left(x_{k}^{(n)}(0)-D_{k}^{(n)}(t)+A_{k}^{(n)}(t)\right)$. Replacing in (30), we get that $x_{k}^{(n)}(t) \geq x_{k}^{(n)}(0)-D_{k}^{(n)}(t)+$ $A_{k}^{(n)}(t)-\left(x_{k}^{(n)}(0)-D_{k}^{(n)}(t)+A_{k}^{(n)}(t)\right)=0$, therefore the scaled queue lengths are kept nonnegative. We now focus on the cases where $x_{k}^{(n)}(t)$ tends to become negative if no reflection was present. Let $t_{1}$ the smallest time instance where $x_{k}^{(n)}\left(t^{-}\right)=0$ such that $x_{k}^{(n)}\left(t_{1}\right)<0$, that is when the process of scaled queue lengths hits zero with a direction to get negative. Therefore $x_{k}^{(n)}(0)+A_{k}^{(n)}\left(t_{1}\right)-D_{k}^{(n)}\left(t_{1}\right)<0$. Then, for $t<t_{1}, z_{k}^{(n)}(t)=0$ and for $t=t_{1}$ we have $z_{k}^{(n)}\left(t_{1}\right)=-\left(x_{k}^{(n)}(0)+A_{k}^{(n)}\left(t_{1}\right)-D_{k}^{(n)}\left(t_{1}\right)\right)>0$, therefore the process increases the first time $x_{k}^{(n)}(t)$ hits zero with a direction to get negative. Now consider some time instance $t_{i}>t_{1}$. Then we will have $z_{k}^{(n)}(t)=-\min _{t_{1} \leq s \leq t}\left\{x_{k}^{(n)}(0)+A_{k}^{(n)}(s)-D_{k}^{(n)}(s)\right\}$. If $x_{k}^{(n)}\left(t_{i}\right)>0$ then $x_{k}^{(n)}(0)-D_{k}^{(n)}\left(t_{i}\right)+A_{k}^{(n)}\left(t_{i}\right)>$ $\min _{t_{1} \leq s \leq t_{i}}\left\{x_{k}^{(n)}(0)+A_{k}^{(n)}(s)-D_{k}^{(n)}(s)\right\}, \quad$ therefore $z_{k}^{(n)}($.$) does not increase. On the other hand, if at$ $t_{i}$ the scaled queue length process (with reflection term up to but not including $t_{i}$ tends to become negative there is $x_{k}^{(n)}(0)-D_{k}^{(n)}\left(t_{i}\right)+A_{k}^{(n)}\left(t_{i}\right)<$ $\min _{t_{1} \leq s<t_{i}}\left\{x_{k}^{(n)}(0)+A_{k}^{(n)}(s)-D_{k}^{(n)}(s)\right\}$, therefore $z_{k}^{(n)}($.$) increases to -\left(x_{k}^{(n)}(0)-D_{k}^{(n)}\left(t_{i}\right)+A_{k}^{(n)}\left(t_{i}\right)\right)$ (it is 
an increase because in this case the expression inside the minimization is negative). For the cases where $x_{k}^{(n)}(t)$ hits the barrier at zero but immediately after it turns positive, the reflection does not change. We have thus shown that the reflection term (36) keeps indeed the queue lengths nonnegative and can increase only when the queue length hits zero.

Following the discussion in subsections $\mathrm{A}$ and $\mathrm{B}$, as all terms in the above expression converge weakly as $n \rightarrow$ $\infty, z_{k}^{(n)}(t) \stackrel{w}{\rightarrow} z_{k}(t)$ accordingly, and actually becomes the amount of data that could have been transmitted in the asymptotic system if the queue was not empty at time $t$.

\section{.4 Further Analysis of the Equation}

So far we have shown that under heavy traffic conditions a properly scaled version of the queue lengths converges weakly to

$\mathbf{x}(t)=\mathbf{x}(0)-\int_{0}^{t} \mathbf{f}(\mathbf{u}(s)) d s+\boldsymbol{\Sigma}_{a} \mathbf{w}_{a}(t)+\boldsymbol{\Sigma}_{d} \mathbf{w}_{d}(t)+\mathbf{z}(t)$.

By assumption, the arrival processes are independent of the channel processes so the corresponding Wiener processes are independent. Therefore, we have $\boldsymbol{\Sigma}_{a} \mathbf{w}_{a}(t)+$ $\boldsymbol{\Sigma}_{d} \mathbf{w}_{d}(t)=\boldsymbol{\Sigma} \mathbf{w}(t)$, where $\mathbf{w}(t)$ is a standard $K$ dimentional Wiener process and $\boldsymbol{\Sigma}$ satisfying (9).

\section{REFERENCES}

[1] A. Destounis, M. Assaad, M. Debbah, B. Sayadi, and A. Feki, "A heavy traffic approach for queue-aware power control over interfering wireless links," in Proc. SPAWC 2012, Jun. 2012.

[2] - "Heavy traffic asymptotic approach for video streaming over small cell networks with imperfect state information," in 28th meeting WWRF, Apr. 2012.

[3] J. Hoydis, M. Kobayashi, and M. Debbah, "Green small-cell networks," IEEE Veh. Technol. Mag., vol. 6, no. 1, pp. 37 -43, Mar. 2011.

[4] M. Karakayali, G. Foschini, and R. Valenzuela, "Network coordination for spectrally efficient communications in cellular systems," IEEE Trans. Wireless Commun., vol. 13, no. 4, pp. $56-61$, Aug. 2006.

[5] D. Gesbert, S. Hanly, H. Huang, S. Shamai Shitz, O. Simeone, and W. Yu, "Multi-cell mimo cooperative networks: A new look at interference," IEEE Journal on Selected Areas in Communications, vol. 28, no. 9, pp. $1380-1408$, Dec. 2010.

[6] M. Chiang, P. Hande, T. Lan, and C. W. Tan, "Power control in wireless cellular networks," Found. Trends Netw., vol. 2, pp. 381533, Apr. 2008

[7] H. Dahrouj and W. Yu, "Coordinated beamforming for the multicell multi-antenna wireless system," IEEE Trans. on Wireless Commun., vol. 9, no. 5, pp. 1748 -1759, May 2010.

[8] G. Foschini and Z. Miljanic, "A simple distributed autonomous power control algorithm and its convergence," IEEE Trans. Veh. Technol., vol. 42, no. 4, pp. 641 -646, Nov. 1993.

[9] S. Lakshminarayana, M. Assaad, and M. Debbah, "H-infinity control based scheduler for the deployment of small cell networks," in Proc. WiOpt2011, May 2011, pp. 9 -16.

[10] Y. Huang and S. Mao, "Downlink power control for variable bit rate videos over multicell wireless networks," in INFOCOM, 2011 Proceedings IEEE, Apr. 2011, pp. 2561 -2569.

[11] H. Huang and V. Lau, "Delay-optimal user scheduling and intercell interference management in cellular network via distributive stochastic learning," IEEE Trans. Wireless Commun., vol. 9, no. 12, pp. $3790-3797$, Dec. 2010.

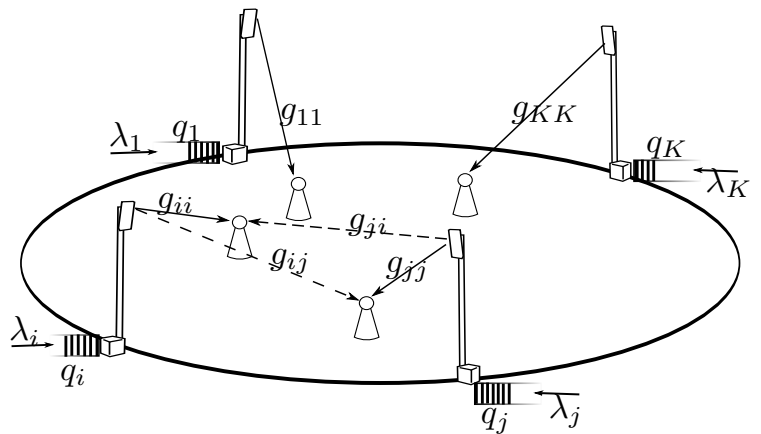

Fig. 1: Illustration of the system model

[12] Y. Cui, V. Lau, R. Wang, H. Huang, and S. Zhang, "A survey on delay-aware resource control for wireless systems: Large deviation theory, stochastic lyapunov drift, and distributed stochastic learning," IEEE Trans. Inf. Theory, vol. 58, no. 3, pp. $1677-1701$, Mar. 2012.

[13] L. Georgiadis, M. J. Neely, and L. Tassiulas, "Resource allocation and cross-layer control in wireless networks," Found. Trends Netw., pp. 1-144, Apr 2006.

[14] H. J. Kushner, Heavy Traffic Analysis of Controlled Queuing and Communication Networks. Berlin and New York: Springrer-Verlag, 2001.

[15] A. L. Stolyar, "Maxweight scheduling in a generalized switch: State space collapse and workload minimization in heavy traffic," The Annals of Applied Probability, vol. 14, no. 1, pp. 1-53, 2004.

[16] S. Shakkottai, R. Srikant, and A. L. Stolyar, "Pathwise optimality of the exponential scheduling rule for wireless channels," $A d-$ vances in Applied Probability, vol. 36, no. 4, pp. 1021-1045, 2004.

[17] S. Bhardwaj, R. J. Williams, and A. S. Acampora, "On the performance of a two-user mimo downlink system in heavy traffic," IEEE Trans. Inf. Theory, vol. 53, no. 5, pp. 1851 -1859, May 2007.

[18] S. Bhardwaj and R. Williams, "Diffusion approximation for a heavily loaded multi-user wireless communication system with cooperation," Queuing Syst., vol. 62, pp. 345-382, 2009.

[19] W. Dai, "Optimal Rate Scheduling via Utility-Maximization for J-User MIMO Markov Fading Wireless Channels with Cooperation," ArXiv e-prints, Jun. 2011.

[20] R. Buche and H. J. Kushner, "Control of mobile communications with time-varying channels in heavy traffic," IEEE Trans. Autom. Control, vol. 47, no. 6, pp. $992-1003$, Jun. 2002.

[21] R. T. Buche and C. Lin, "Heavy traffic control policies for wireless systems with time-varying channels," in Proc. ACC 2005, Jun. 2005, pp. $3972-3974$ vol. 6.

[22] W. Wu, A. Arapostathis, and S. Shakkottai, "Optimal power allocation for a time-varying wireless channel under heavy-traffic approximation," IEEE Trans. Autom. Control, vol. 51, no. 4, pp. 580 - 594, Apr. 2006.

[23] R. Berry and R. Gallager, "Communication over fading channels with delay constraints," IEEE Trans. Inf. Theory, vol. 48, no. 5, pp. 1135-1149, May 2002.

[24] H. S. Wang and N. Moayeri, "Finite-state markov channel-a useful model for radio communication channels," IEEE Trans. Veh. Technol., vol. 44, no. 1, pp. 163 -171, Feb. 1995.

[25] D. Tse and P. Viswanath, Fundamentals of Wirless Communications, 2005.

[26] A. R. Ward and P. W. Glynn, "Properties of the Reflected Ornstein Uhlenbeck Process,," Queueing Systems, vol. 44, pp. 109-123, 2003.

[27] S. Lakshminaryana, J. Hoydis, M. Debbah, and M. Assaad, "Asymptotic analysis of distributed multi-cell beamforming," in Proc. IEEE PIMRC, Sept. 2010.

[28] C. W. Gardiner, Handbook of stochastic methods : for physics, chemistry and the natural sciences, ser. Springer series in synergetics, 13. Springer, 2002. 


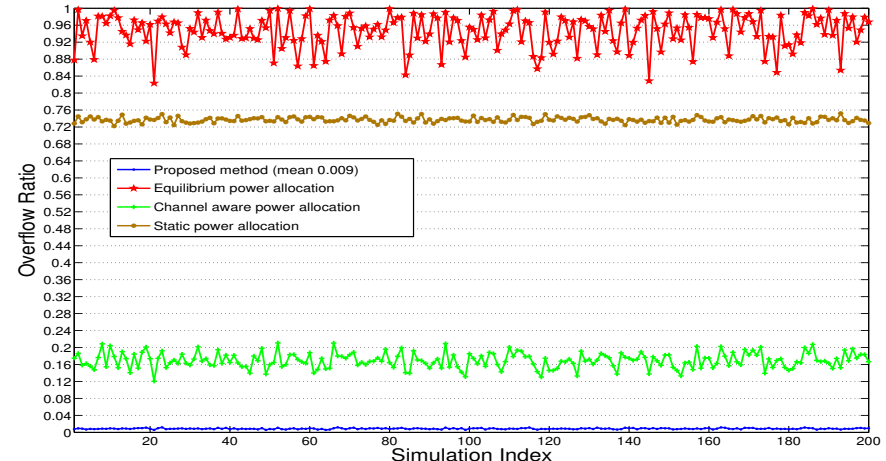

Fig. 2: Overflow ratios for the queue at link 1 in the thee links setting.

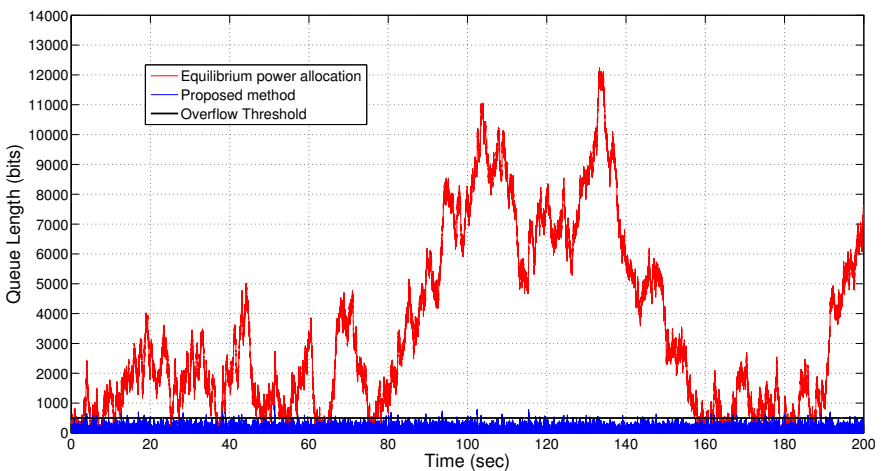

Fig. 3: Evolution of the queue lengths at transmitter 1 in the 3 links setting in one simulation run, using the equilibrium and the proposed power allocation method.

\begin{tabular}{|l|}
\hline \\
PLACE \\
PHOTO \\
HERE \\
\end{tabular}

Apostolos Destounis obtained the Diploma degree in Electrical and Computer Engineering from National Technical University of Athens in 2009 and the M.Sc. degree in Communications and Signal Processing from Imperial College London in 2010. Since 2011 he is with AlcatelLucent Bell Labs France and the Alcatel Lucent Chair on Flexible radio at Supelec working towards a Ph.D. on service aware resource alocation in wireless networks.

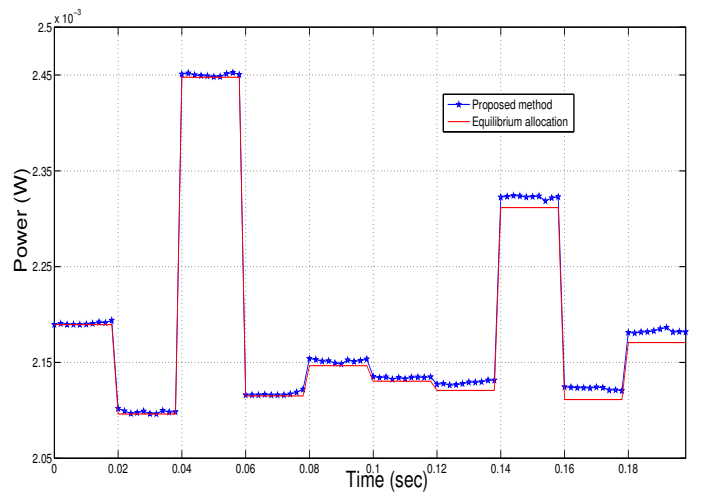

Fig. 4: Evolution of the equilibrium and allocated power using our method for Link 1 for the first 100 timeslots of a simulation

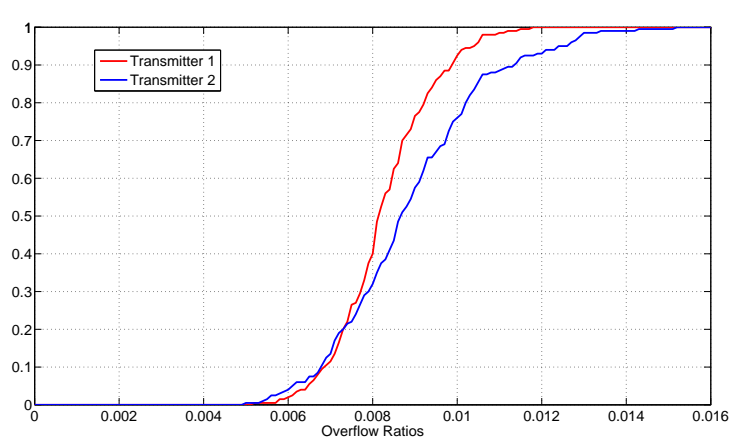

Fig. 5: CDF over the 200 simulation runs of the overflow ratios for 2 transmitter-receiver pairs and 3 antennas per transmitter.

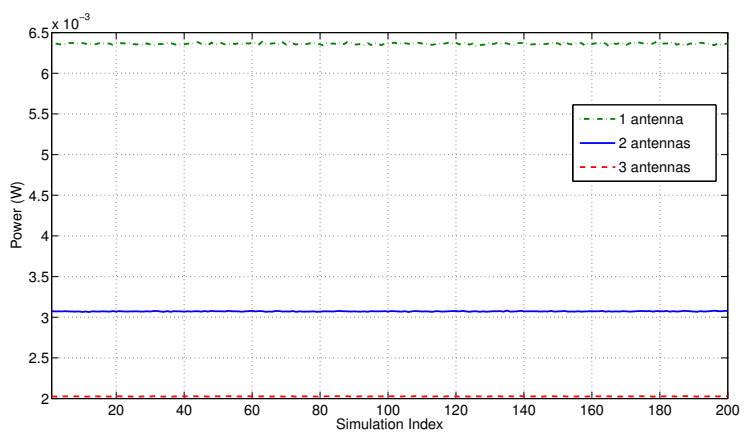

Fig. 6: Average power usage for the 2 transmitter-receiver pair scenario and different number of antennas at the tansmitters

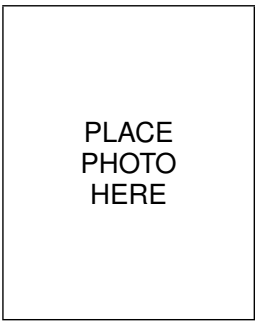

Mohamad Assaad received the B.E. degree in electrical engineering with high honors from Lebanese University, Beirut, in 2001. He received the M.Sc. and Ph.D. degrees (with high honors), both in telecommunications, from Ecole Nationale Superieure des Telecommunications (ENST), Paris, France, in 2002 and 2006, respectively. While pursuing the Ph.D. degree, he was a Research Assistant with the Wireless Networks and Multimedia Services Department, INT, France, working on cross-layer design in UMTS/HSDPA system and interaction of TCP with MAC/RLC and physical layers. Since March 2006, he has been with the Telecommunications Department, SUPELEC, where he is currently an Associate Professor. His research interests include cross-layer design, resource optimization in wireless networks and multi antenna systems.

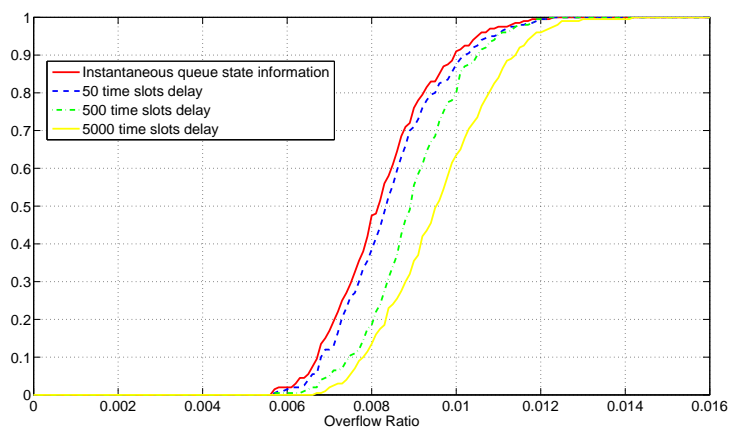

Fig. 7: CDFs over the 200 simulation runs of the overflow ratios for the proposed algorithm implemented with delayed information or Link 1 


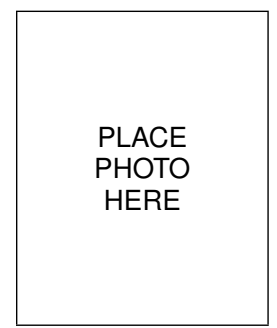

Mérouane Debah entered the Ecole Normale Suprieure de Cachan (France) in 1996 where he received his M.Sc and Ph.D. degrees respectively. He worked for Motorola Labs (Saclay, France) from 1999-2002 and the Vienna Research Center for Telecommunications (Vienna, Austria) until 2003. He then joined the Mobile Communications department of the Institut Eurecom (Sophia Antipolis, France) as an Assistant Professor until 2007. He is now a Full Professor at Supelec (Gif-sur-Yvette, France), holder of the Alcatel-Lucent Chair on Flexible Radio and a recipient of the ERC starting grant MORE (Advanced Mathematical Tools for Complex Network Engineering). His research interests are in information theory, signal processing and wireless communications. He is a senior area editor for IEEE Transactions on Signal Processing. Mrouane Debbah is the recipient of the "Mario Boella" award in 2005, the 2007 General Symposium IEEE GLOBECOM best paper award, the Wi-Opt 2009 best paper award, the 2010 Newcom++ best paper award as well as the Valuetools 2007, Valuetools 2008, Valuetools 2012 and CrownCom2009 best student paper awards. He is a WWRF fellow. In 2011, he received the IEEE Glavieux Prize Award.

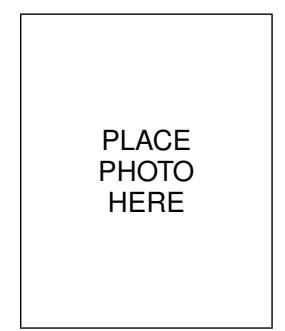

Bessem Sayadi is a member of technical staff in Multimedia Technology domain at Alcatel-Lucent Bell Labs. He is member of Alcatel-Lucent Technical Academy, since 2008. He received M.Sc. (00) and Ph.D. (03) degrees in Control and Signal processing from Suplec, Paris-Sud University, with highest distinction. He worked previously as a postdoctoral fellow in the National Centre for Scientific Research (CNRS), and as a senior researcher engineer in Orange Labs. His main research interests are in the area of broadcast technology (DVB, 3GPP), video coding and transport, and resource allocation algorithms for communication networks. He has authored over 50 publications in journal and conference proceedings and serves as a regular reviewer for several technical journals and conferences. He holds 7 patents and has more than twenty patent applications pending in the area of video coding and wireless communications.

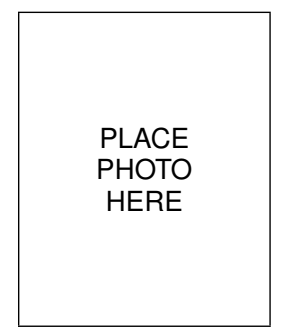

Afef Feki is a research engineer at Alcatel Lucent Bell Labs, France. She holds an engineering degree in telecommunications as well as an M.Sc. in optics and radio frequency, both from the Polytechnic Institute of Grenoble in France, and she received a Ph.D. in telecommunications from Telecom ParisTech with high honours. Her research interests at Bell Labs include 4G wireless networks, femto and small cells deployment, and green networks, as well as self configuration and management of radio networks.

Previously, she worked at Orange Labs R\&D France where she was involved in various projects on cognitive radio, dynamic spectrum access issues, and the optimization of radio resource management. She has published over 10 journal and conference papers, and holds several patents. She is co-chair of the International Workshop on Indoor and Outdoor Femto Cells (IOFC), TPC co-chair of WiOpt 2012, and TPC member for other conferences such as WCNC 2012 and VTC 2011. 